\title{
Percolative Electrical Conductivity of Platy Alumina/Few-layer Graphene Multilayered Composites
}

\author{
Ki-Beom Choi, Jong-Young Kim ${ }^{\dagger}$, Sung-Min Lee, Kyu-Hyoung Lee*, and Dae Ho Yoon** \\ Icheon Branch, Korea Institute of Ceramic Engineering and Technology, Icheon 17303, Korea \\ *Department of Nano Applied Engineering, Kangwon National University, Chuncheon 24341, Korea \\ **Department of Materials Science and Engineering, Sungkyunkwan University, Suwon 16419, Korea
}

(Received April 15, 2017; Accepted May 15, 2017)

\begin{abstract}
In this work, we present a facile one-pot synthesis of a multilayer-structured platy alumina/few-layer graphene nanocomposite by planetary milling and hot pressing. The sintered composites have electrical conductivity exhibiting percolation behavior (threshold $\sim 0.75$ vol.\%), which is much lower than graphene oxide/ceramic composites (>3.0 vol.\%). The conductivity data are well-described by the percolation theory, and the fitted exponent values are estimated to be 1.65 and 0.93 for $t$ and $q$, respectively. The $t$ and $q$ values show conduction mechanisms intermediate between 2D- and 3D, which originates from quantum tunneling between nearest neighbored graphenes.
\end{abstract}

Key words : Ceramic, Graphene, Percolation, Electrical conductivity

\section{Introduction}

$\mathbf{F}$ abrication of graphene-insulator composites are attracting much attention for potential applications in chargestorage capacitors, ${ }^{1)}$ gate-dielectrics in field effect transistors ${ }^{2)}$ and sensors. ${ }^{3)}$ The composites are promising not only for their percolative M-I transition but also for their dramatic increase in the dielectric constant near the percolation threshold. The percolative M-I transition and abnormal increase in the dielectric constant of ceramic/metallic composites have also generated interest, ${ }^{4-7)}$ but they are not practically applied owing to their high metal loading, high dielectric loss, or unstable properties near the threshold. Recently, due to the outstanding electrical and mechanical properties of carbon nanotubes (CNTs) and graphene sheets, the fabrication of graphene- or CNT-ceramic composites has attracted enthusiastic attention..$^{8-13)}$ A low percolation threshold in graphene- and CNT-composites was achieved by a high aspect ratio in graphene layers and CNTs and their high electrical conductivity.

In this work, we increased the electrical conductivity of alumina ceramics by embedding graphene nanosheets between alumina $\left(\mathrm{Al}_{2} \mathrm{O}_{3}\right)$ layers. We fabricated multi-layered alumina/few-layer graphene (FLG) composites with anisotropically oriented plate-like grains by mechanical milling and hot pressing. We found that electrical conductivity exhibits percolation behaviour with a threshold value of 0.75 vol.\% with embedded graphene layers in an alumina

\footnotetext{
${ }^{\dagger}$ Corresponding author: Jong-Young Kim

E-mail : jykim@kicet.re.kr

Tel : +82-31-645-1423 Fax : +82-31-645-1486
}

matrix. According to the percolation theory, the critical exponents ( $t$ and $q$ ) were extracted by fitting the conductivity data with respect to the graphene concentration. Such conducting ceramic/graphene composites are promising for use in highly conducting electroceramics (conducting parts in CVD, chemical vapor deposition, and etcher equipment) and antistatic ceramics for electronic and communication devices.

\section{Experimental Procedure}

\subsection{Platy Alumina/FLG Composite Synthesis}

First, $10 \mathrm{~g}$ of platy alumina powder (>99.0\%, 160SG, Merck, Germany) was added to $65 \mathrm{~mL}$ of N-Methyl-2-pyrrolidone (NMP, Daejung, Korea). Then, FLG powder (3 nm, SKU-NP, Graphene supermarket, USA) was added to the alumina slurry. To obtain a homogeneous suspension, the aqueous slurry was milled by a planetary mill (Pulverisette 5 , Fritch, Germany) using alumina balls $(\varphi=3.0 \mathrm{~mm})$ as a medium for $10 \mathrm{~h}$ with a speed of $250 \mathrm{rpm}$ as shown in Fig. 1. The ball to powder weight ratio was $4: 1$. The milled slurry was dried in a vacuum oven at $200^{\circ} \mathrm{C}$ for $24 \mathrm{~h}$. The dried powder was sintered by hot pressing (Hot press furnace, Thermotech, Korea) in an Ar flowing atmosphere. The sintering temperature was raised at a rate of $10^{\circ} \mathrm{C} / \mathrm{min}$ and maintained at $1500^{\circ} \mathrm{C}$ for $1 \mathrm{~h}$ under a pressure of $30 \mathrm{MPa}$ (Fig. 1).

\subsection{Characterization}

The microstructure of the sintered samples was investigated using field-emission electron microscopy, FE-SEM (JSM-6710F, Jeol, Japan). The microstructure of the com- 


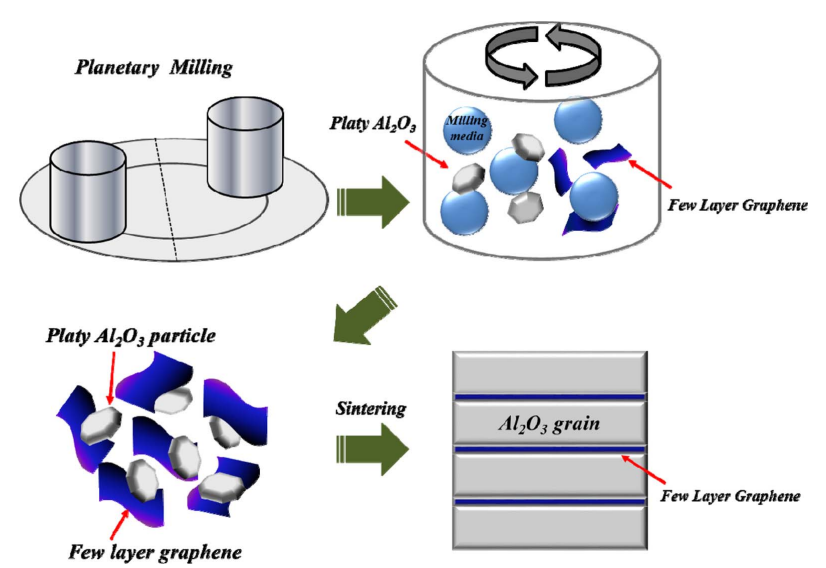

Fig. 1. Schematic of the synthesis of platy alumina/few-layer graphene (FLG) composite by planetary milling in a wet medium.

posites were investigated using transmission electron microscopy, TEM (JEM-4010, Jeol, Japan).

\subsection{Electrical conductivity Measurement}

The volume resistivity was measured at a constant voltage of $1 \mathrm{~V} / \mathrm{mm}$ with a source meter (617, Keithley, United states) using the guarded electrode method according to ASTM D257 association standards.

\section{Results and Discussion}

\subsection{Synthesis of Platy Alumina/FLG Composite by Planetary Milling}

Recently, ball milling techniques employing higher mechanical energy such as planetary milling using a medium (DMF, $\mathrm{CO}_{2}$, melamine, salt) ${ }^{14-17)}$ and stirred media milling using smaller media ${ }^{18,19)}$ has been found to be effective to produce graphenes. The high mechanical energy of planetary milling is favorable for the exfoliation of graphite, while its drawbacks are long processing time (several tens of hours) and a post-dispersing step using sonication due to low yield. To obtain the hybrid powders, we adopted a planetary milling technique to allow pulverization and uniform mixing of FLG powder (Fig. 2(a)) and platy alumina (Fig. 2(b)) using NMP as a dispersing media. Then, the acquired powders were compacted into bulk composites by hot press, and we investigated the electrical and dielectric properties of the composites.

The anisotropic structure of the platy alumina/FLG composites was derived by using platy alumina instead of spherical alumina as a matrix. We fabricated self-assembled solids with a multilayer dielectric/graphene structure composed of platy alumina/FLG with enhanced permittivity. We synthesized multi-layered structures using platy alumina (thickness $=300-400 \mathrm{~nm}$, lateral dimension $>10$ $\mu \mathrm{m})$ and FLG (thickness $\sim 3 \mathrm{~nm}$, lateral dimension $>5 \mu \mathrm{m}$ ). The final layer thickness values in the sintered platy alumina/FLG composites were determined by Field-emission Electron microscopy (FE-SEM) and Transmission Electron Microscopic (TEM) analysis. As shown in Figs. 2(c) and 2(d), the platy alumina layer thickness obtained by FE-SEM analysing was $0.3-1 \mu \mathrm{m}$, whereas the FLG layer thickness obtained by TEM analysis was $3-5 \mathrm{~nm}$. The dielectric prop-

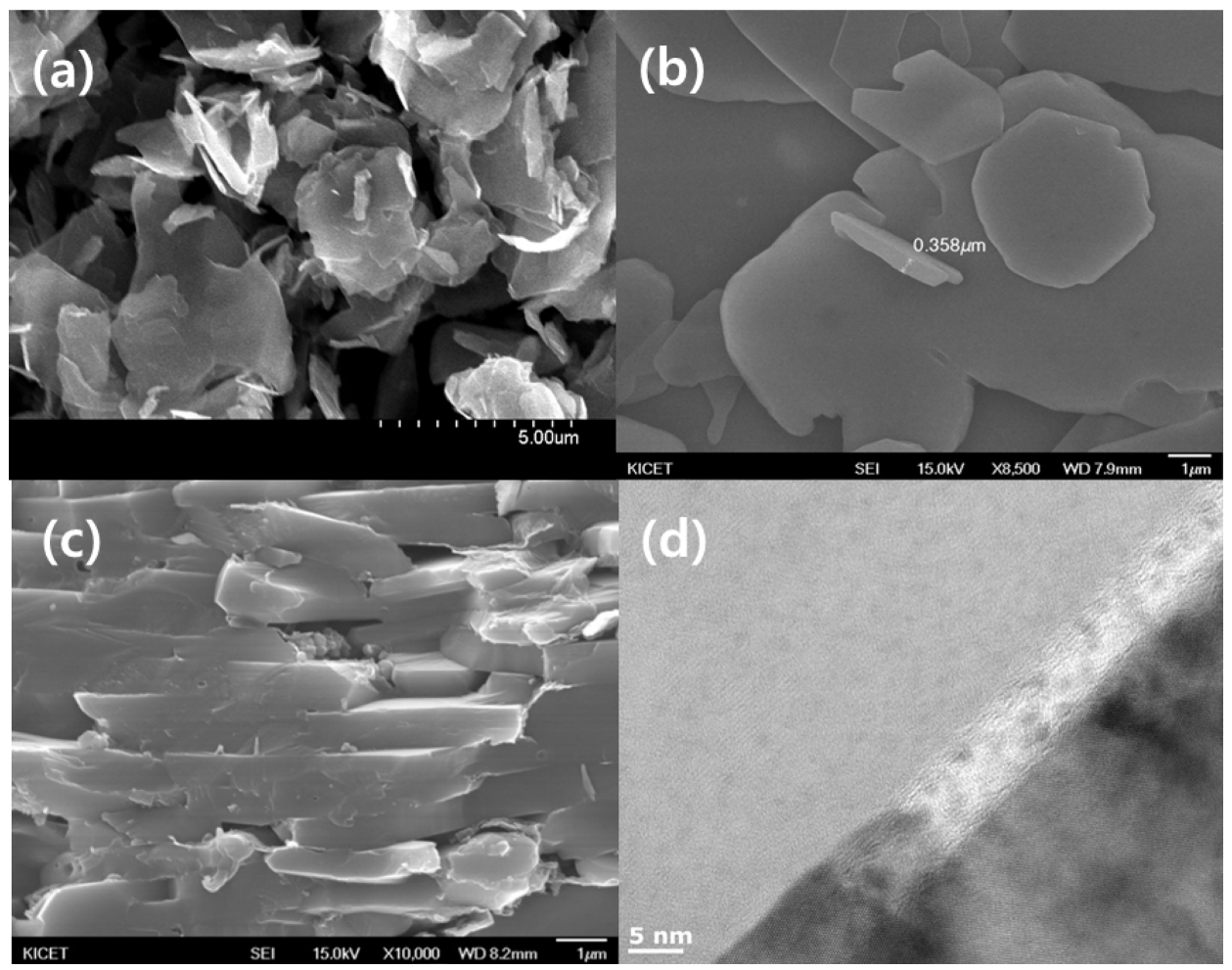

Fig. 2. SEM image of (a) FLG, (b) platy alumina, (c) 0.299 vol.\% FLG/alumina, (d) TEM image of FLG in (c). 
Table 1. Electrical Resisitivity of Platy Alumina/FLG Composites

\begin{tabular}{ccccccccccc}
\hline FLG VOL\% & 0.276 & 0.299 & 0.564 & 0.718 & 0.741 & 0.798 & 0.815 & 0.905 & 0.936 & 1.631 \\
\hline Resistivity $(\Omega \mathrm{cm})$ & $1.3 \mathrm{E} 14$ & $1.3 \mathrm{E} 14$ & $1.2 \mathrm{E} 13$ & $1.2 \mathrm{E} 13$ & $2.4 \mathrm{E} 13$ & $3.1 \mathrm{E} 3$ & $5.9 \mathrm{E} 2$ & $6.3 \mathrm{E} 1$ & $1.2 \mathrm{E} 2$ & $1.6 \mathrm{E} 1$ \\
\hline
\end{tabular}

erty in the direction parallel to the hot pressing pressure was measured by impedance spectroscopy.

\subsection{Electrical Properties of the Platy alumina/FLG Composite}

The electrical resistivity values of the composites at room temperature are shown in Table 1 . According to the resistivity versus FLG concentration, below $\sim 0.75$ vol.\% of FLG, the resistivity decreased slowly with an increasing FLG concentration, while the resistivity abruptly decreased above $\sim 0.75$ vol. $\%$ of FLG (Fig. 3). This phenomena can be explained by the metal-insulator (MI) transition by the percolation theory. ${ }^{5-7)}$ In our composites, the percolation threshold was 0.7 - 0.8 vol.\% and the MI transition occurred in this region, which enhanced the electrical conduction by several orders of magnitude. The percolation threshold of the FLG/ platy alumina composite was only 0.75 vol.\%, which is comparable to the experimental values for $\mathrm{GO} / \mathrm{spherical}$ alumina $\left(f_{c}=0.38 \text { vol. } \%\right)^{13)}$ and MWCNT/alumina $\left(f_{c}=0.79\right.$ vol.\%). ${ }^{20)}$ This value is much lower than the GO/silica composite $(\sim 3.9 \text { wt. } \%)^{21)}$ and FLG/spherical alumina $\left(f_{c}=3.0\right.$ vol.\%). ${ }^{12)}$ The calculated threshold value of the composite with ellipsoids and an aspect ratio of 100 - 1000 ranged from 1.3 to 0.13 vol. $\%{ }^{22)}$ therefore, our threshold value for FLG with a thickness of $<5 \mathrm{~nm}$ and a lateral dimension of $2-3$ $\mu \mathrm{m}$ was quite consistent with a well-dispersed disk-shaped filler (FLG) in a dielectric alumina matrix.

According to the percolation theory, the electrical conductivity of the composite material containing a metallic filler (M) in a dielectric matrix (D) is given by the power law as follows. ${ }^{23)}$

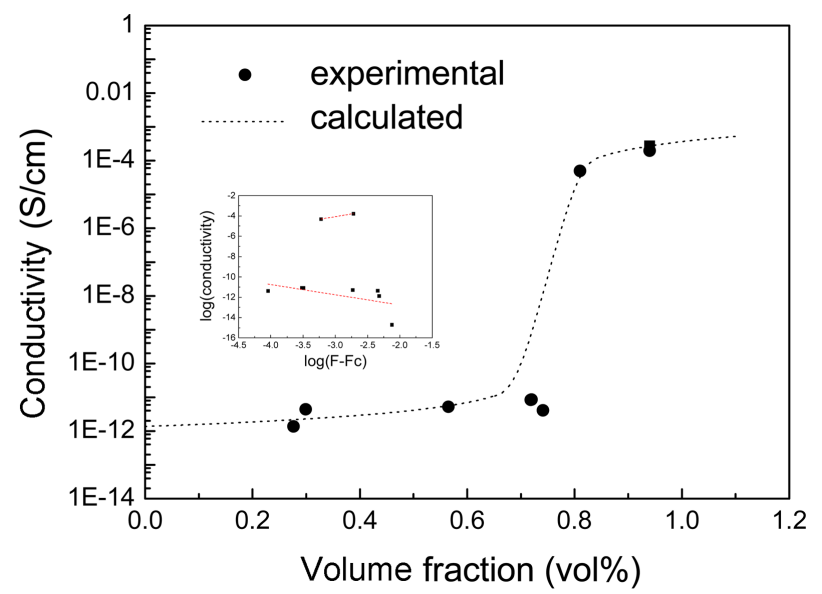

Fig. 3. Electrical conductivity of platy alumina/FLG composites as a function of the FLG volume fraction. Experimental data were fitted by the calculated curve according to Equations (1) and (2).

$$
\begin{aligned}
& \langle\sigma\rangle=\sigma_{M}\left(f-f_{c}\right)^{\mathrm{t}} f>f_{c} \\
& \langle\sigma\rangle=\sigma_{D}\left(f_{c}-f\right)^{-q} f<f_{c}
\end{aligned}
$$

where $\sigma_{D}$ : the conductivity of the dielectric material, $\sigma_{M}$ : the conductivity of the metallic filler, $f$ : the volume fraction of the dielectric and conducting filler, $q=$ the critical exponent before the percolation threshold, and $t=$ the critical exponent after the percolation threshold.

For the plotting log (conductivity) vs. the log $\left(f-f_{c}\right)$ linearly, we calculate the $\sigma_{D}, \sigma_{M}, t$, and $q$ values. The $f$ is the volume fraction of the FLGs, $f_{c}$ is the critical volume fraction or percolation threshold, and the exponent $t$ reflects the dimensionality of the system. The fitted values according to equations (1) and (2) are quite consistent with the experimental values (Fig. 3). These parameters are given in Table 2 . The fitted $t$ value of 1.65 in the proposed system is similar to the reported values for mullite-molybdenum cermet $(t=$ $1.6)^{7)}$ and alumina-FLG composites $(t=1.84) .{ }^{13)}$ In the percolation theory based on a discrete lattice-percolation network, the $t$ values are 1.3 and 2.0 in $2 \mathrm{D}$ - and 3D-systems, respectively. ${ }^{24)}$

The conductivity below the percolation threshold is also described by equation (2). We fitted this equation to the data for the composites and obtained a value of $q=0.929$ (Fig. 3). This value is slightly lower than $t=1.1-1.3$ for a $2 \mathrm{D}$-system and comparable to $q=0.7-1.0$ for a 3D-system. ${ }^{24)}$

In classical percolation theory, the conducting network is considered to be established by the connection between the neighboring conducting fillers. However, in some cases, the competition between percolative conduction and tunneling between conducting particles is suggested..$^{25,26)}$ Although the conducting particles are not geometrically connected, electrical conduction occurs via tunneling. In such cases, a nonuniversal critical exponent $t$ as high as 5 - 10 has been observed in percolative composites. ${ }^{25,27)}$ In our study, $t$ and $q$ values were intermediate between those for the $2 \mathrm{D}$-systme and the 3D- system. This might be because of non-negligible quantum tunneling between neighboring graphenes. However, the graphenes are separated by at least $>1.0 \mathrm{~mm}$ by platy alumina partcles with regular thickness (Fig. 1) and, therefore, the interdistance distribution of the graphenes are not quite as broad as randomly distributed graphenes in previously reported polymer composites.

Table 2. Fitted Parameters by Equations (1) and (2) from Electrical Resisitivity of the Composites

\begin{tabular}{ccccc}
\hline$\sigma_{D}(\mathrm{~S} / \mathrm{cm})$ & $\sigma_{M}(\mathrm{~S} / \mathrm{cm})$ & $f_{c}$ & $q$ & $t$ \\
\hline $1.0 \mathrm{E}-16$ & 200 & 0.0075 & 0.929 & 1.65 \\
\hline
\end{tabular}




\section{Conclusions}

We demonstrated a facile, scalable, one-pot synthesis of ceramics/FLG composites by planetary ball milling. The use of NMP as dispersants for platy alumina and FLG effectively produced well-dispersed slurry of a platy alumina/ FLG composite. The electrical conductivity of the composites exhibited percolative behavior with a low percolation threshold because of the high aspect ratio of FLG. The proposed FLG/platy alumina composite is promising for electrically conducting electroceramics.

\section{Acknowledgements}

This work was financially supported by the Ministry of Trade, Industry and Energy (10053585).

\section{REFERENCES}

1. J.-Y. Kim, W. H. Lee, J. W. Suk, J. R. Potts, H. Chou, I. N. Kholmanov, R. D. Piner, J. Lee, D. Akinwande, and R. S. Ruoff, "Chlorination of Reduced Graphene Oxide Enhances the Dielectric Constant of Reduced Graphene Oxide/Polymer Composites," Adv. Mater., 25 [16] 2308-13 (2013).

2. I. Meric, M. Y. Han, A. F. Young, B. Ozyilmaz, P. Kim, and K. L. Shepard, "Current Saturation in Zero-Bandgap, TopGated Graphene Field-Effect Transistors," Nat. Nanotechnol., 3 [11] 654-59 (2008).

3. C. Liu, Z. Yu, D. Neff, A. Zhamu, and B. Z. Jang, "GrapheneBased Supercapacitor with an Ultrahigh Energy Density," Nano Lett., 10 [12] 4863-68 (2010).

4. H. Chang, Z. Sun, K. Y. F. Ho, X. Tao, F. Yan, W. M. Kwok, and Z. Zheng, "A Highly Sensitive Ultraviolet Sensor Based on a Facile in situ Solution-Grown ZnO Nanorod/Graphene Heterostructure," Nanoscale, 3 [1] 258-64 (2011).

5. S. Kirkpatrick, "Percolation and Conduction," Rev. Mod. Phys., 45 [4] 574-88 (1973).

6. B. I. Halperin, S. Feng, and P. N. Sen, "Differences between Lattice and Continuum Percolation Transport Exponents," Phys. Rev. Lett., 54 [22] 2391-94 (1985).

7. C. Pecharroman and J. S. Moya, "Experimental Evidence of a Giant Capacitance in Insulator-Conductor Composites at the Percolation Threshold," Adv. Mater., 12 [4] 294-97 (2000).

8. C. Ramirez, F. M. Figueiredo, P. Miranzo, P. Poza, and M. I. Osendi, "Graphene Nanoplatelet/Silicon Nitride Composites with High Electrical Conductivity," Carbon, 50 [10] 3607-15 (2012).

9. C. Ramirez, L. Garzon, P. Miranzo, M. I. Osendi, and C. Ocal, "Electrical Conductivity Maps in Graphene Nanoplatelet/Silicon Nitride Composites Using Conducting Scanning Force Microscopy," Carbon, 49 [12] 3873-80 (2011).

10. E. Lee, K. B. Choi, S. M. Lee, J. Y. Kim, J. Y. Jung, S. W. Baik, Y. S. Lim, S. J. Kim, and W. Shim, "A Scalable and Facile Synthesis of Alumina/Exfoliated Graphite Composites by Attrition Milling," RSC Adv., 5 [113] 93267-73 (2015).

11. H. J. Kim, S. M. Lee, Y. S. Oh, Y. H. Yang, Y. S. Lim, D. H. Yoon, C. Lee, J. Y. Kim, and R. S. Ruoff, "Unoxidized Graphene/Alumina Nanocomposite: Fracture- and WearResistance Effects of Graphene on Alumina Matrix," Sci.
Rep., 45176 (2014).

12. Y. Fan, L. Wang, J. Li, J. Li, S. Sun, F. Chen, L. Chen, and W. Jiang, "Preparation and Electrical Properties of Graphene Nanosheet $/ \mathrm{Al}_{2} \mathrm{O}_{3}$ Composites," Carbon, 48 [6] 1743-49 (2010).

13. Y. Fan, W. Jiang, and A. Kawasaki, "Highly Conductive FewLayer Graphene $/ \mathrm{Al}_{2} \mathrm{O}_{3}$ Nanocomposites with Tunable Charge Carrier Type," Adv. Funct. Mater., 22 [18] 3882-89 (2012).

14. W. Zhao, M. Fang, F. Wu, H. Wu, L. Wang, and G. Chen, "Preparation of Graphene by Exfoliation of Graphite Using Wet Ball Milling," J. Mater. Chem., 20 [28] 5817-19 (2010).

15. I. Y. Jeon, Y. R. Shin, G. J. Sohn, H. J. Choi, S. Y. Bae, J. Mahmood, S. M. Jung, J. M. Seo, M. J. Kim, D. W. Chang, L. Dai, and J. B. Baek, "Edge-Carboxylated Graphene Nanosheets via Ball Milling," Proc. Natl. Acad. Sci. U. S. A., 109 [15] 5588-93 (2012).

16. V. Leon, A. M. Rodriguez, P. Prieto, M. Prato, and E. Vazquez, "Exfoliation of Graphite with Triazine Derivatives under Ball-Milling Conditions: Preparation of FewLayer Graphene via Selective Noncovalent Interactions," ACS Nano, 8 [1] 563-71 (2014).

17. Y. Lv, L. Yu, C. Jiang, S. Chen, and Z. Nie, "Synthesis of Graphene Nanosheet Powder with Layer Number Control via a Soluble Salt-Assisted Route," RSC Adv., 4 [26] 1335054 (2014)

18. C. Damm, T. J. Nacken, and W. Peukert, "Quantitative Evaluation of Delamination of Graphite by Wet Media Milling," Carbon, 81 284-94 (2015).

19. C. Knieke, A. Berger, M. Voigt, R. N. K. Taylor, J. Rohrl, and W. Peukert, "Scalable Production of Graphene Sheets by Mechanical Delamination," Carbon, 48 [11] 3196-204 (2010).

20. K. Ahmad, W. Pan, and S. L. Shi, "Electrical Conductivity and Dielectric Properties of Multiwalled Carbon Nanotube and Alumina Composites," Appl. Phys. Lett., 89 [13] 133122 (2006).

21. S. Watcharotone, D. A. Dikin, S. Stankovich, R. Piner, I. Jung, G. H. B. Dommett, G. Evmenenko, S.-E. Wu, S.-F. Chen, C. Liu, S. T. Nguyen, and R. S. Ruoff, "Graphene-Silica Composite Thin Films as Transparent Conductors," Nano Lett., 7 [7] 1888-92 (2007).

22. E. J. Garboczi, K. A. Snyder, J. F. Douglas, and M. F. Thorpe, "Geometrical Percolation Threshold of Overlapping Ellipsoids," Phys. Rev. E, 52 [1] 819-28 (1995).

23. A. L. Efros and B. I. Shklovskii, "Critical Behaviour of Conductivity and Dielectric Constant near the Metal-Non-Metal Transition Threshold," Phys. Status Solidi B, 76 [2] 475-85 (1976).

24. D. Stauffer and A. Aharony, Introduction to Percolation Theory; pp. 93-121, Tayler and Francis, London, 1994.

25. Z. Rubin, S. A. Sunshine, M. B. Heaney, I. Bloom, and I. Balberg, "Critical Behavior of the Electrical Transport Properties in a Tunneling-Percolation System," Phys. Rev. B, 59 [19] 12196-99 (1999).

26. P. Sheng, E. K. Sichel, and J. I. Gittleman, "FluctuationInduced Tunneling Conduction in Carbonpolyvinylchloride Composites," Phys. Rev. Lett., 40 [18] 1197-200 (1978).

27. G. Cunningham, M. Lotya, N. McEvoy, G. S. Duesberg, P. van der Schoot, and J. N. Coleman, "Percolation Scaling in Composites of Exfoliated $\mathrm{MoS}_{2}$ Filled with Nanotubes and Graphene," Nanoscale, 4 [20] 6260-64 (2012). 